Case Report

\title{
Unusual Presentation of Unilateral Isolated Probable Lyme Optic Neuritis
}

\author{
Ahmet Z. Burakgazi ${ }^{1}$ and Carl S. Henderson ${ }^{2}$ \\ ${ }^{1}$ Internal Medicine, Neurology Department, Virginia Tech Carilion School of Medicine and Research Institute, Roanoke, \\ VA 24016, USA \\ ${ }^{2}$ Internal Medicine, Rheumatology Department, Virginia Tech Carilion School of Medicine and Research Institute, Roanoke, \\ VA 24016, USA \\ Correspondence should be addressed to Ahmet Z. Burakgazi; drburakgazi@yahoo.com
}

Received 14 August 2015; Revised 4 January 2016; Accepted 10 January 2016

Academic Editor: Chin-Chang Huang

Copyright (C) 2016 A. Z. Burakgazi and C. S. Henderson. This is an open access article distributed under the Creative Commons Attribution License, which permits unrestricted use, distribution, and reproduction in any medium, provided the original work is properly cited.

\begin{abstract}
Optic neuritis $(\mathrm{ON})$ is one of the most common manifestations of central nervous system involvement caused by various etiologies. Lyme $\mathrm{ON}$ is an exceedingly rare ocular manifestation of Lyme disease (LD) and only a few cases have been published in the literature. Lyme $\mathrm{ON}$ is very rare but should be included in the differential diagnosis in unexplained cases, particularly in Lyme endemic areas. Careful and detailed examination and investigation are warranted to make the diagnosis. We report this case to increase awareness of clinicians to include Lyme disease in differential diagnosis of $\mathrm{ON}$ for unexplained cases of $\mathrm{ON}$. Herein we present a unique case with a unilateral $\mathrm{ON}$ caused by LD along with pre- and posttreatment findings and literature review.
\end{abstract}

\section{Introduction}

Optic neuritis (ON) is an inflammation of the optic nerve and one of the most common manifestations of central nervous system involvement caused by various etiologies [15]. Well-descried causes of optic neuritis include demyelinating diseases $[4,5]$, autoimmune diseases [2-4], inflammatory diseases, infections, and vaccinations. The cardinal signs of ON are decreased visual acuity, a central visual field defect, dyschromatopsia, and a relative afferent pupil defect [2-5].

Lyme disease (LD), the leading arthropod-borne infection in the northern hemisphere, can cause a wide spectrum of neurological conditions involving central and peripheral nervous systems [6-8]. LD-related ocular complications are uncommon, but various types of manifestations have been described including conjunctivitis, keratitis, and extra-ocular muscle palsies $[8,9]$. Lyme $\mathrm{ON}$ is an exceedingly rare ocular manifestation of LD and only a few cases have been published in the literature.
Herein, we report a unique case with a unilateral ON caused by LD along with pre- and posttreatment findings and literature review.

\section{Case Presentation}

A 59-year-old female with a recent past medical history of mesentery thrombosis on stable anticoagulation and minimal other past medical history was referred to our clinic from ophthalmology clinic for unexplained decreased right eye visual acuity and visual field defect and right optic disc swelling. She presented with approximately $3-4$ weeks history of right eye vision changes and seen by rheumatologist after she was noted to have a positive ANA. She had right blurry vision that worsened with focusing on near objects. One to two weeks after having vision symptoms, she noticed papular erythematous blanching rash across the shoulders extending to midback, part of chest, left shoulder, and right cheek. She also described tiredness and generalized joints pain. She denied any other neurological or systemic symptoms. She did 


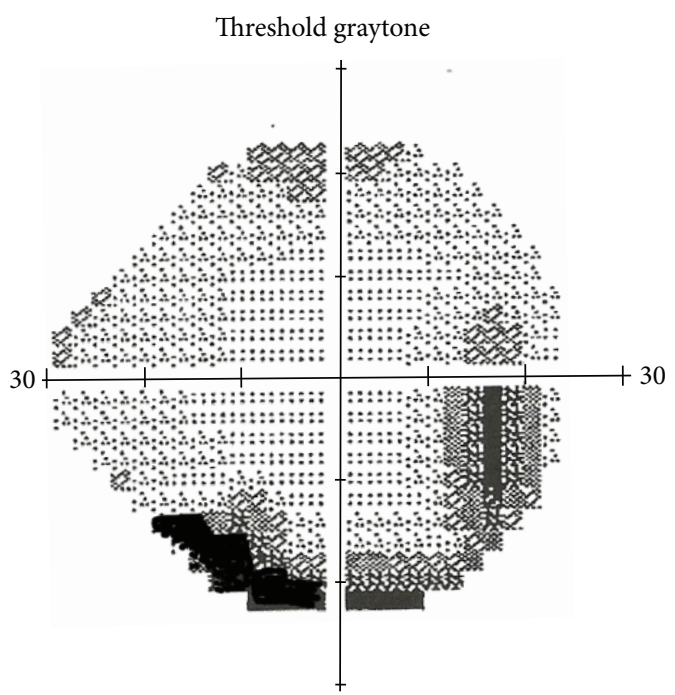

FIGURE 1: An automated perimetry visual field test before the treatment demonstrated arcuate scotoma, inferior partial arcuate defect, and superior vertical step defect that indicated optic nerve fiber bundle involvement.

not recall any tick bite. She did not have similar episodes in the past. She did not have any other medical diseases. She did not take any other medications before her symptoms and any medications on regular basis. Her family history was unremarkable.

Her physical examination showed optic nerve swelling on the right eye and mild afferent pupil defect on the right eye. Visual acuity with Snellen Eye Test Charts was 20/30 on the right eye and 20/20 with the left eye. The remainder of her neurological examination, including cranial nerves, motor exam, sensory exam, reflexes, coordination, and gait, was within normal limits. No meningeal signs such as neck stiffness were detected.

She underwent detailed investigation including biochemical tests, magnetic resonance imaging (MRI) of brain, magnetic resonance venography (MRV) of head, and lumbar puncture. The cerebrospinal fluid examination showed normal cell count (white blood cell count: 4 ; and red blood cell count: 3 ), mildly elevated protein $55 \mathrm{mg} / \mathrm{dL}$ (normal range $15-45 \mathrm{mg} / \mathrm{dL}$ ), elevated Lyme disease IgG 1:32 (reference ranges IgG $<1: 4$ ), and positive oligoclonal bands ( $>5$ well defined gamma restriction bands). She underwent extensive blood tests which showed positive Lyme Western Blot with bands (positive seven of $10 \mathrm{IgG}$ bands; positive three of three IgM bands); and normal results for other tests including Creactive protein, sedimentation rate, antinuclear antibodies, 14-3-3 eta protein, C3 complement, C4 complement, total complement, CCP Antibody, rheumatoid factor, quantiferonTB, HIV 1,2 AB, Hepatitis C antibody, Hepatitis B surface antigen, Hepatitis B surface antibody, cardiolipin antibodies, beta-s glycoprotein 1 antibodies $\operatorname{IgA} / \operatorname{IgM}$, and lupus anticoagulant panel. An automated perimetry visual field test before the treatment demonstrated arcuate scotoma, inferior partial arcuate defect, and superior vertical step defect that indicated optic nerve fiber bundle involvement (Figure 1).

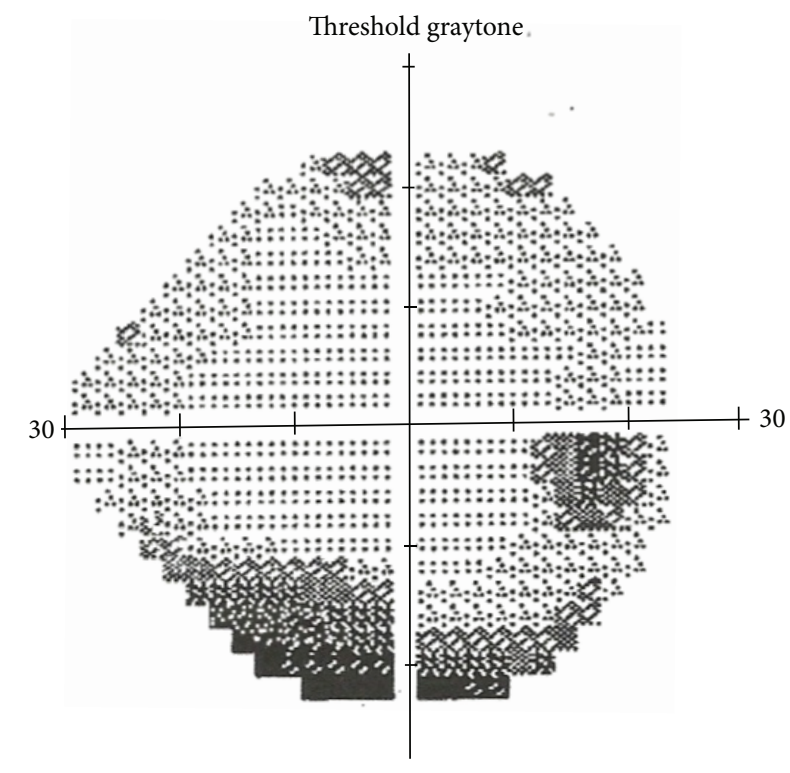

FIGURE 2: The follow-up perimetry visual field test after sixweek doxycycline treatment demonstrated improvements of arcuate scotoma, superior vertical step, and inferior partial arcuate defects.

She was consulted to dermatology for the rash. The biopsy suggested granuloma annulare, in an interstitial pattern as seen with infections such as LD.

She was treated with doxycycline $100 \mathrm{mg}$ twice a day for 6 weeks. She noticed some degree of improvement in her vision at the middle of the treatment, and she felt significant recovery at the end of the treatment. With the treatment, she felt more energy, less tiredness, and less joint pain. The followup ophthalmological examination showed decreased optic disc edema and improved visual acuity. Visual acuity with Snellen Eye Test Charts was 20/20 on the right eye and 20/20 with the left eye. The follow-up perimetry visual field test after six-week doxycycline treatment demonstrated improvements of arcuate scotoma, superior vertical step, and inferior partial arcuate defects (Figure 2).

\section{Discussion}

In this report, we present a case with isolated unilateral Lyme $\mathrm{ON}$ that is an extremely rare complication of LD. In our case, anti-Borrelia antibodies were present in serum and CSF and favorable outcome was achieved with six-week treatment of doxycycline. Further investigations were performed to rule out other possible etiologies of optic neuritis before making the final diagnosis. The patient also had some vague symptoms including arthralgia and skin rash that improved with doxycycline treatment.

Lyme $\mathrm{ON}$ is a rare complication of $\mathrm{LD}[10]$. A few cases of Lyme ON have been reported in the literature. Blanc et al. reported two cases of isolated Lyme ON. Both patients had positive serum and cerebrospinal fluid serology, a positive intrathecal anti-Borrelia antibody index, and a good outcome on ceftriaxone [11]. Scott et al. reported a 10-year-old girl presenting with chiasmal ON whose history, clinical course, 
and increased serum Lyme immunoglobulin G titer were consistent with Lyme disease that improved with antibiotic treatment [12]. The last case of Lyme ON was reported in 2010. Although few isolated Lyme ON cases were reported in the literature, Kubová et al's cohort study including 81 patients with neuroborreliosis showed $42 \%$ had blurred vision or diplopia and $27 \%$ had a delayed visual evoked potential that means Lyme related optic nerve involvement may be more common than previously assumed [13]. We encountered this unusual presentation of $\mathrm{ON}$, and all other etiological reasons were ruled out before making the final diagnosis of Lyme ON.

Ceftriaxone, cefotaxime, penicillin $\mathrm{G}$, and doxycycline are highly effective in most neurological Lyme cases $[6,7$, 14-17]. Intravenous ceftriaxone, high-dose penicillin, and cefotaxime have been used in patients with severe disease, particularly nervous system infection, and in patients who have not responded to oral regimens. European studies $[6,14$, $15,18-22]$ have shown that oral doxycycline, which achieves spirochetocidal concentration in the central nervous system (CNS), is highly effective in Lyme meningitis, cranial neuritis, and radiculoneuritis. In our case, the patient had a favorable improvement with oral doxycycline treatment; most of the reported Lyme ON cases were treated with ceftriaxone $[11,12$, $16,17,23,24]$.

The other unique part of our case was a demonstration of changes in visual field test before and after doxycycline treatment. We showed some improvements in visual field test with six-week doxycycline treatment. Thus, the visual field test can be used to assess the effectiveness of antibiotic treatment in Lyme ON.

A causal link between Lyme seropositivity and isolated $\mathrm{ON}$ is controversial. A few conflictive studies are available to discuss the relationship between LD and ON [24, 25]. Jacobson et al. [25] investigated 20 consecutive patients with newly diagnosed isolated optic neuritis who resided in a region endemic for $\mathrm{LD}$; four patients had positive serum serology for LD. Three of these patients underwent CSF analysis and two had CSF lymphocytic pleocytosis that remained unexplained after extensive evaluations for causes other than LD. Two patients with CSF pleocytosis were treated with intravenous ceftriaxone and the other two received oral antibiotic treatment. Four of the patients had favorable recovery with the treatments [25]. In another study, Sibony et al. [24] retrospectively reviewed Lyme serology in 440 patients with ON examined between 1993 and 2003. They demonstrated that Lyme enzyme-linked immunosorbent assay (ELISA) was positive in $28(6.4 \%)$ patients with optic neuritis, three of whom had syphilis with cross-reactive antibodies. Of the remaining 25 ELISA-positive patients, only one had Lyme disease-related optic neuritis and the other 24 cases had reactive Lyme serologies related to a history of LD years earlier, asymptomatic exposure, false-positive results, or nonspecific humoral expansion. Western blots confirmatory tests were negative in those 24 cases with reactive Lyme serologies. It was concluded that there is insufficient evidence for a causal link between LD and ON or neuroretinitis [24]. In our case, comprehensive investigation was performed to rule out other more common causes of optic neuritis [2$5,11,23,26]$. After ruling out the other more common reason, we included Lyme disease in the differential diagnosis.

\section{Conclusion}

In conclusion, $\mathrm{ON}$ is one of the most common manifestations of nervous system involvement caused by several autoimmune, inflammatory, and infection diseases. Lyme $\mathrm{ON}$ is rare but should be included in the differential diagnosis in unexplained cases, particularly in Lyme endemic areas. Careful and detailed examination and investigation are warranted to make the diagnosis. We report this case to increase awareness of clinicians to include Lyme disease in differential diagnosis of $\mathrm{ON}$ for unexplained cases of $\mathrm{ON}$.

\section{Conflict of Interests}

The authors declare that there is no conflict of interests.

\section{References}

[1] R. W. Beck and R. L. Gal, "Treatment of acute optic neuritis: a summary of findings from the optic neuritis treatment trial," Archives of Ophthalmology, vol. 126, no. 7, pp. 994-995, 2008.

[2] G. S. Hoffman, G. S. Kerr, R. Y. Leavitt et al., "Wegener granulomatosis: an analysis of 158 patients," Annals of Internal Medicine, vol. 116, no. 6, pp. 488-498, 1992.

[3] M. J. Kupersmith, R. M. Burde, F. A. Warren, T. G. Klingele, L. P. Frohman, and H. Mitnick, "Autoimmune optic neuropathy: evaluation and treatment," Journal of Neurology, Neurosurgery and Psychiatry, vol. 51, no. 11, pp. 1381-1386, 1988.

[4] A. Malik, M. Ahmed, and K. Golnik, "Treatment options for atypical optic neuritis," Indian Journal of Ophthalmology, vol. 62, no. 10, pp. 982-984, 2014.

[5] J. Sellner, M. Boggild, M. Clanet et al., "EFNS guidelines on diagnosis and management of neuromyelitis optica," European Journal of Neurology, vol. 17, no. 8, pp. 1019-1032, 2010.

[6] A. Z. Burakgazi, "Lyme disease-induced polyradiculopathy mimicking amyotrophic lateral sclerosis," International Journal of Neuroscience, vol. 124, no. 11, pp. 859-862, 2014.

[7] J. J. Halperin, E. L. Logigian, M. F. Finkel, and R. A. Pearl, "Practice parameters for the diagnosis of patients with nervous system Lyme borreliosis (Lyme disease)," Neurology, vol. 46, no. 3, pp. 619-627, 1996.

[8] J. J. Halperin, D. J. Volkman, and P. Wu, "Central nervous system abnormalities in Lyme neuroborreliosis," Neurology, vol. 41, no. 10, pp. 1571-1582, 1991.

[9] J. J. Halperin, B. J. Luft, A. K. Anand et al., "Lyme neuroborreliosis: central nervous system manifestations," Neurology, vol. 39, no. 6, pp. 753-759, 1989.

[10] F. Träisk and L. Lindquist, "Optic nerve involvement in Lyme disease," Current Opinion in Ophthalmology, vol. 23, no. 6, pp. 485-490, 2012.

[11] F. Blanc, L. Ballonzoli, C. Marcel, S. De Martino, B. Jaulhac, and J. de Seze, "Lyme optic neuritis," Journal of the Neurological Sciences, vol. 295, no. 1-2, pp. 117-119, 2010.

[12] I. U. Scott, A. Silva-Lepe, and R. M. Siatkowski, "Chiasmal optic neuritis in lyme disease," American Journal of Ophthalmology, vol. 123, no. 1, pp. 136-138, 1997.

[13] Z. Kubová, J. Szanyi, J. Langrová, J. Kremláček, M. Kuba, and K. Honegr, "Motion-onset and pattern-reversal visual evoked 
potentials in diagnostics of neuroborreliosis," Journal of Clinical Neurophysiology, vol. 23, no. 5, pp. 416-420, 2006.

[14] T. Lapp, "AAP issues recommendations on the prevention and treatment of Lyme disease," American Family Physician, vol. 61, no. 11, pp. 3463-3464, 2000.

[15] H.-W. Pfister, V. Preac-Mursic, B. Wilske, and K. M. Einhaupl, "Cefotaxime vs penicillin $\mathrm{G}$ for acute neurologic manifestations in Lyme borreliosis. A prospective randomized study," Archives of Neurology, vol. 46, no. 11, pp. 1190-1194, 1989.

[16] J. J. Halperin, E. D. Shapiro, E. Logigian et al., "Practice parameter: treatment of nervous system Lyme disease (an evidencebased review): report of the Quality Standards Subcommittee of the American Academy of Neurology," Neurology, vol. 69, no. 1, pp. 91-102, 2007.

[17] G. P. Wormser, R. J. Dattwyler, E. D. Shapiro et al., "The clinical assessments treatment, and prevention of lyme disease, human granulocytic anaplasmosis, and babesiosis: clinical practice guidelines by the Infectious Diseases Society of America," Clinical Infectious Diseases, vol. 43, no. 9, pp. 1089-1134, 2006.

[18] J. J. Halperin, E. L. Logigian, M. F. Finkel, and R. A. Pearl, "Practice parameters for the diagnosis of patients with nervous system Lyme borreliosis (Lyme disease)," Neurology, vol. 46, no. 3, pp. 619-627, 1996.

[19] S. W. Luger, P. Paparone, G. P. Wormser et al., "Comparison of cefuroxime axetil and doxycycline in treatment of patients with early Lyme disease associated with erythema migrans," Antimicrobial Agents and Chemotherapy, vol. 39, no. 3, pp. 661-667, 1995.

[20] D. Bremell and L. Dotevall, "Oral doxycycline for Lyme neuroborreliosis with symptoms of encephalitis, myelitis, vasculitis or intracranial hypertension," European Journal of Neurology, vol. 21, no. 9, pp. 1162-1167, 2014.

[21] U. Ljøstad, E. Skogvoll, R. Eikeland et al., "Oral doxycycline versus intravenous ceftriaxone for European Lyme neuroborreliosis: a multicentre, non-inferiority, double-blind, randomised trial," The Lancet Neurology, vol. 7, no. 8, pp. 690-695, 2008.

[22] A. Mygland, U. Ljostad, V. Fingerle et al., "EFNS guidelines on the diagnosis and management of European Lyme neuroborreliosis," European Journal of Neurology, vol. 17, no. 1, pp. 8-16, el-e4, 2010.

[23] C. Burkhard, M. Gleichmann, and H. Wilhelm, "Optic nerve lesion following neuroborreliosis: a case report," European Journal of Ophthalmology, vol. 11, no. 2, pp. 203-206, 2001.

[24] P. Sibony, J. Halperin, P. K. Coyle, and K. Patel, "Reactive Lyme serology in optic neuritis," Journal of Neuro-Ophthalmology, vol. 25, no. 2, pp. 71-82, 2005.

[25] D. M. Jacobson, J. J. Marx, and A. Dlesk, "Frequency and clinical significance of Lyme seropositivity in patients with isolated optic neuritis," Neurology, vol. 41, no. 5, pp. 706-711, 1991.

[26] J. Shimizu, Y. Hatanaka, M. Hasegawa et al., "IFN $\beta$-1b may severely exacerbate Japanese optic-spinal MS in neuromyelitis optica spectrum," Neurology, vol. 75, no. 16, pp. 1423-1427, 2010. 


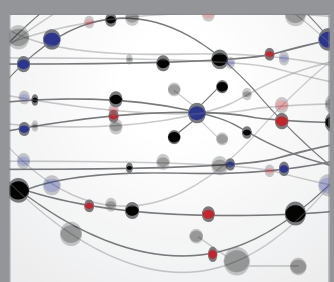

The Scientific World Journal
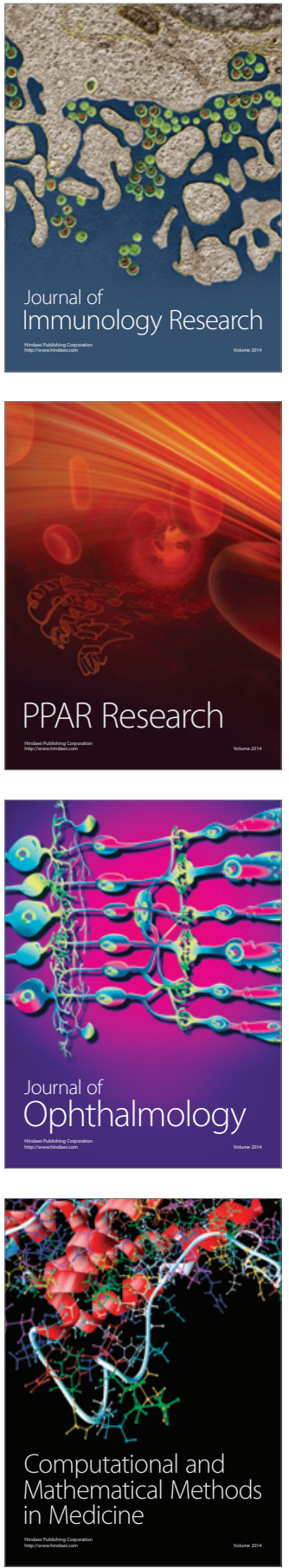

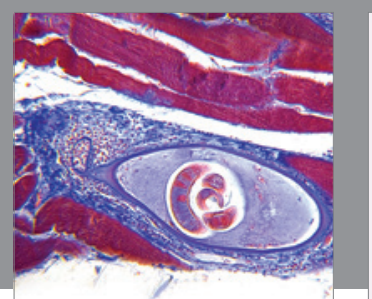

Gastroenterology Research and Practice

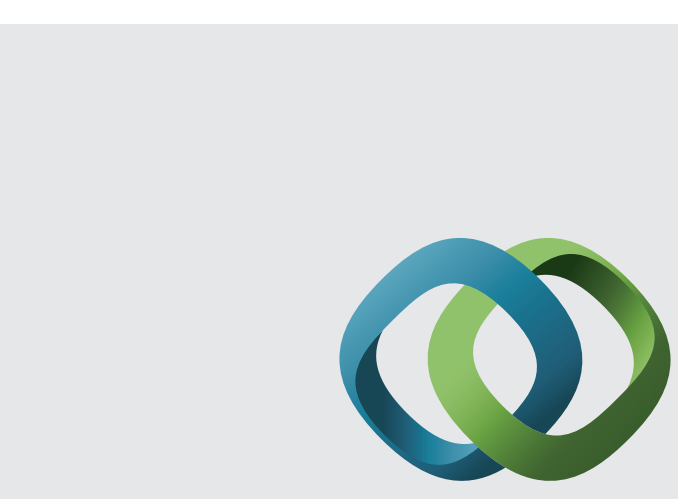

\section{Hindawi}

Submit your manuscripts at

http://www.hindawi.com
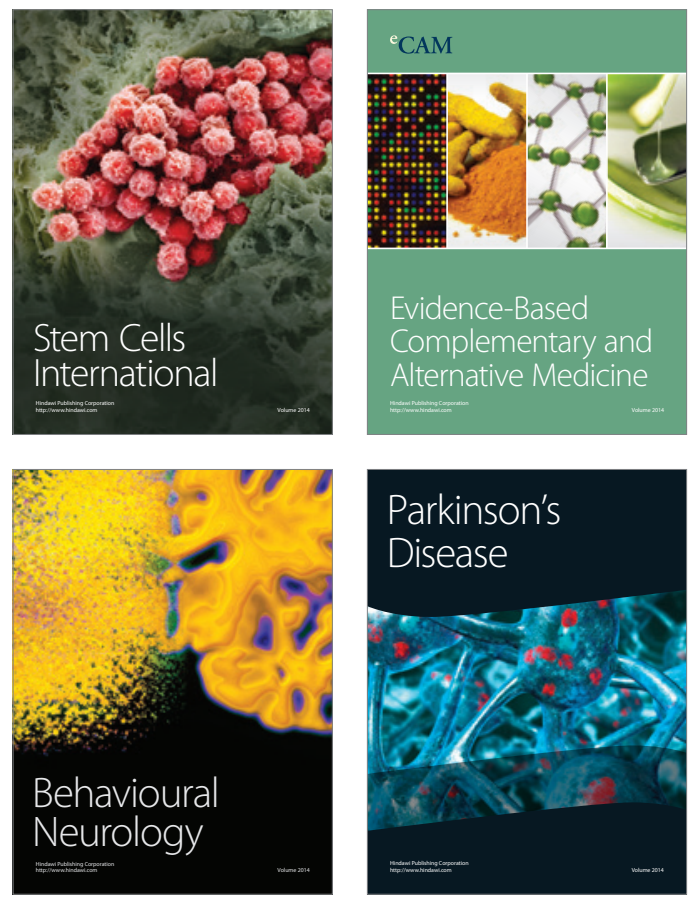
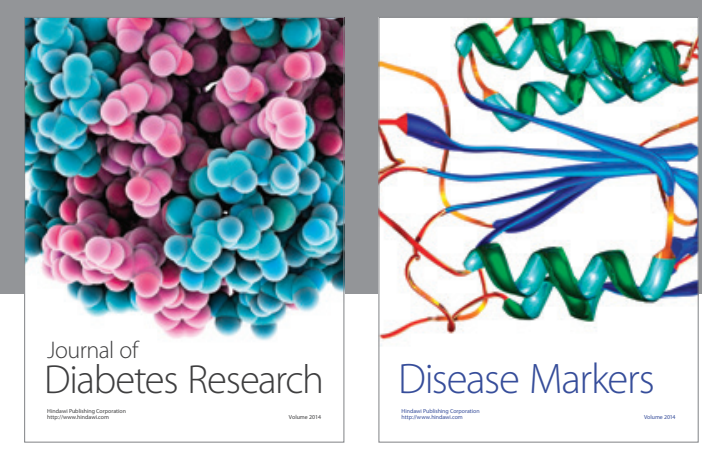

Disease Markers
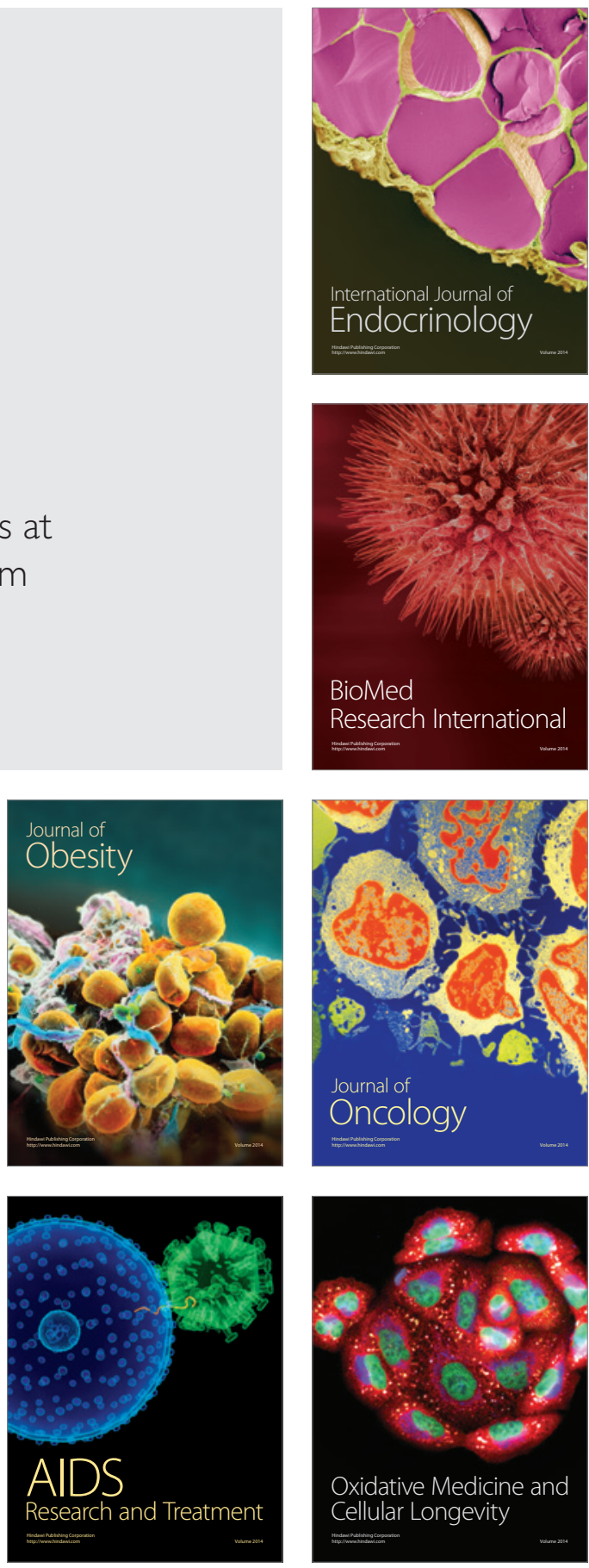